\title{
Low-Dose and High-Speed Observations of Battery Processes by Operando STEM
}

\author{
B. L. Mehdi ${ }^{1,2}$, N. D. Browning ${ }^{1,2}$, J. Lee ${ }^{1}$, H. Amari ${ }^{1}$, N. Johnson ${ }^{1}$, D. Nicholls ${ }^{1}$, A. J. Stevens ${ }^{3}$ \\ ${ }^{1}$ Engineering \& Physical Sciences, University of Liverpool, Liverpool L69 3GH, UK \\ ${ }^{2}$ Physical and Computational Science Directorate, PNNL, Richland, WA 99352, USA \\ ${ }^{3}$ Sivananthan Laboratories, Sensor Analytics, Bolingbrook, IL USA
}

Reactions at the two interfaces between the main components of a battery - anode/electrolyte and cathode/electrolyte - determine the overall energy density and coulombic efficiency of the system as a whole. While Li-ion batteries are currently the standard for many applications, these batteries have their limitations, and as such, there are research efforts progressing worldwide aimed at developing new more efficient batteries - such as Li-metal, Li-air, Li-sulfur, Na-ion and $\mathrm{Zn}$. In all these cases, the initial promising performance of the half-cell displays issues when combined to form a fully operating cell. Side reactions can cause electrolyte breakdown, passivation or corrosion and the formation of a solidelectrolyte-interphase (SEI) layer. In addition, deposition of an excess of metal ions during charging can lead to the formation of dendrites during cycling. Each of these effects is extremely sensitive to the local chemistry/field at the electrode/electrolyte interface and a full understanding of the materials parameters that can lead to better batteries requires the ability to observe all of the dynamic processes taking place at these interfaces during battery operation.

The kinetics of the reactions at the electrode/electrolyte interface can be studied using in-situ electrochemical stages in the (scanning) transmission electron microscope (S/TEM) [1]. Figure 1 shows the results of electrochemical cycling using a typical Li-ion battery electrolyte. In the images, the presence of $\mathrm{Li}$ can be easily observed by the contrast reversal. To make sure that the electron beam does not have any effect on the electrochemistry being performed [2], for these observations the electron beam dose rate is carefully calibrated to be below the electrolyte damage threshold prior to operando cycling (in this case all experiments use a dose $\leq 0.3$ electrons $/ \AA^{2} / \mathrm{s}$ ) [3]. As such, typical beam effects such as the formation of bubbles and/or precipitates from the breakdown of the electrolyte are avoided. The need to keep the beam dose and dose rate low, however, limits the magnification of the image and the resulting resolution of the quantified observations. To increase our ability to study the lithiation process, we must therefore look to ways to increase the speed of imaging while lowering the dose.

One way of achieving lower dose and higher speed, i.e. more efficient sampling of the images, is to use a sub-sampling and inpainting approach [4]. The acquisition of sub-sampled STEM images can be achieved through an external scan generator that randomly hops between lines of the scan at a rate designed to minimize the effects of scan hysteresis [5]. For a highly sub-sampled image $(<10 \%$ of the pixels) the raw image itself is unrecognizable. When we intentionally sub-sample an image using a higher electron dose in the sampled pixels (giving an overall equivalent dose to the sample as there are fewer pixels) we increase the image acquisition speed to achieve the same high resolution. If the dose is further fractionated in a way that we sub-sample to form a basic reconstructed image and then use that reconstruction to fill in the gaps in the data - termed here as adaptive sub-sampling - we can have the same increase in speed but now also increase the resolution of the image for the same dose [6]. The practical application of this method to study battery processes is shown in Figure 2, where the distribution of Li around the anode shown in Figure 1 can be accurately quantified from a sub-sampled set of pixels. The practical aspects of this sub-sampling approach to the study of rapid transient battery phenomena will be discussed in this presentation [7]. 


\section{References:}

[1] M. Gu, L. R. Parent, B. L. Mehdi, R. Unocic, M. McDowell, R. Sacci, W. Xu, J. Connell, P. Xu, P. Abellan, X. Chen, Z. Yaohui, D. Perea, L. Lauhon, J-G. Zhang, J. Liu, N. D. Browning, Y. Cui, I. Arslan, C. Wang. Nano Letters 13, 6106 (2013).

[2] P. Abellán, C. Park, B. L. Mehdi, W. Xu, Y. Zhang, L. R. Parent, M. Gu, I. Arslan, J. Zhang, C. M. Wang, J. E. Evans, N. D. Browning, Nano Letters 14, 1293 (2014)

[3] B. L. Mehdi, E. Nasybulin, J. Qian, C. Park, D. A. Welch, R. Faller, H. Mehta, W. A. Henderson, W. Xu, C. M.Wang, J. E. Evans, J. -G. Zhang, K. T. Mueller, and N. D. Browning, Nano Letters 15, 2168 (2015)

[4] A. Stevens, H. Yang. L. Carin, I. Arslan and N. D. Browning, Microscopy 63, 41-51 (2014)

[5] L. Kovarik, A. Stevens, A. Liyu, N. D. Browning, Applied Physics Letters 109, 164102 (2016)

[6] A. Stevens, L. Luzi, H. Yang, L. Kovarik, B. L. Mehdi, A. Liyu, M. E. Gehm, N. D. Browning, Applied Physics Letters 112, 043104 (2018)

[7] This work was supported by the U.K. Faraday Institution. A portion of the results were obtained using the Environmental Molecular Sciences Laboratory (EMSL), a national scientific user facility sponsored by the Department of Energy's Office of Biological and Environmental Research and located at PNNL. PNNL is a multi-program national laboratory operated by Battelle for the U.S. Department of Energy (DOE) under Contract DE-AC05-76RL01830.

\section{Li Deposition}

\section{Li Dissolution}
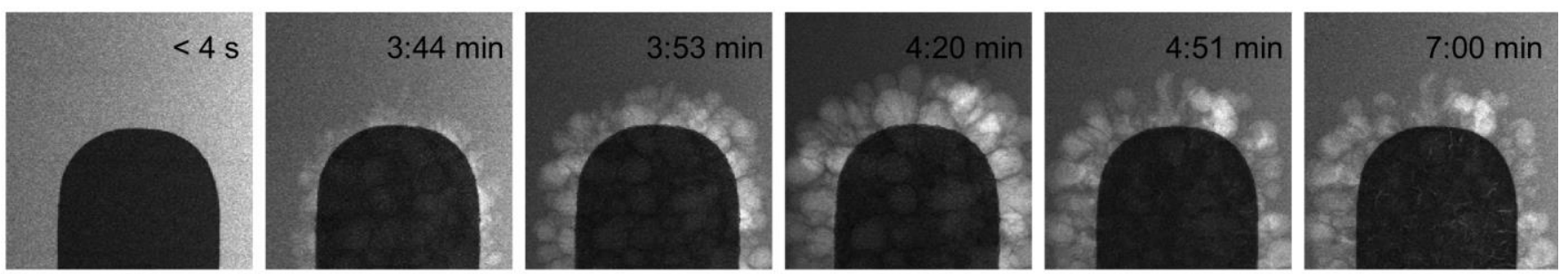

Figure 1: STEM bright field images of Li deposition and dissolution at the interface between a Pt working electrode and the $\mathrm{LiPF}_{6} / \mathrm{PC}$ electrolyte during a charge/discharge cycle of the operando cell.
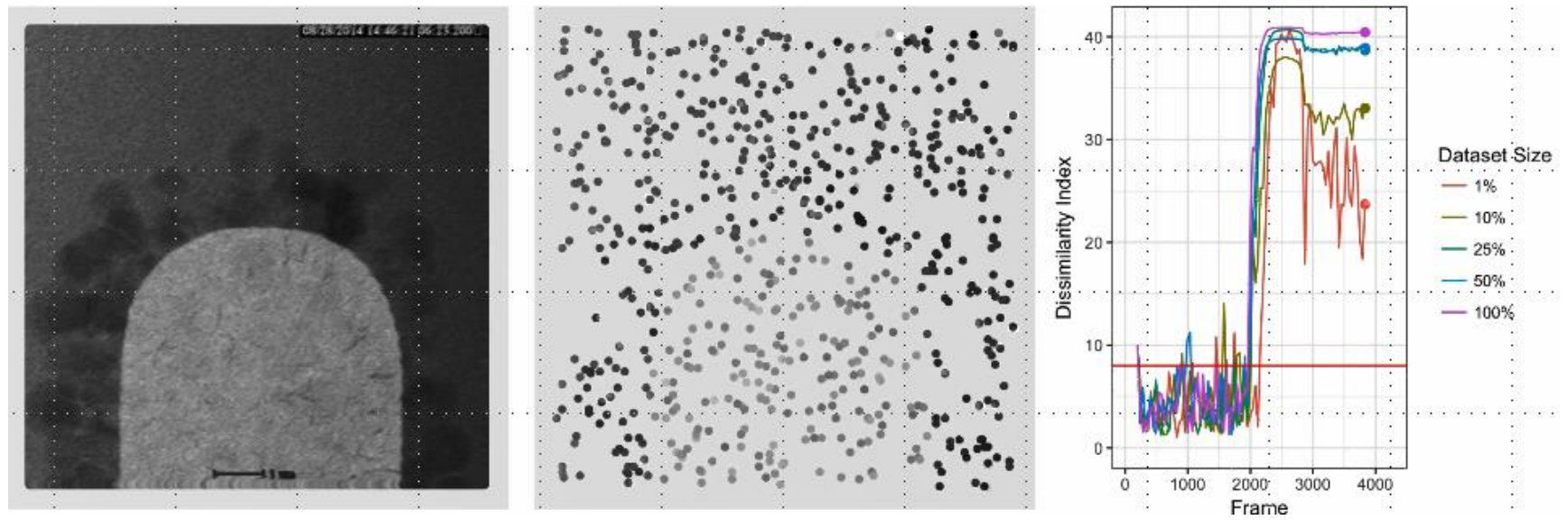

Figure 2: Sub-sampled datasets using only $1 \%$ of the image can provide accurate measurements of the amount of Li deposited during the cycle. 